\title{
Pregnancy and the Control of Epileptic Seizures: A Review
}

\author{
Mervyn J. Eadie
}

Received: March 19, 2021 / Accepted: April 15, 2021 / Published online: May 14, 2021

(C) The Author(s) 2021

\section{ABSTRACT}

Over the past 50 years, published studies have provided quantitative data on the control of epileptic seizures during pregnancy. The studies have varied in quality, and particularly in the ways in which seizure control has been assessed. However, most studies have shown that seizure occurrence rates are more likely to worsen than improve during pregnancy, though in most pregnancies the rates have been unaltered. Nearly all of the studies have involved women with antiseizure medication-treated epilepsy, but there is a little evidence that seizure control also tends to worsen in pregnancies of women with untreated epilepsy. The factors likely to contribute to the seizure worsening are (i) patient non-compliance, (ii) increased antiseizure medication clearance during pregnancy resulting in lower circulating drug concentrations relative to dose, (iii) the effects of the higher female sex hormone levels during pregnancy, oestrogens being pro-epileptogenic and progesterone anti-epileptogenic, and (iv) reluctance to use the potential teratogen valproate in women capable of pregnancy, depriving them of the most effective drug for certain types of epilepsy. Compliance can be encouraged, but at the present time only one other factor is readily correctable, i.e. the increased drug clearance. This can be compensated for by raising antiseizure medication dosage during pregnancy, guided by measurement of circulating drug concentrations. This course of action appears to reduce the chance of seizure disorder worsening during pregnancy, but so far it has not provided a complete solution to the issue.

Keywords: Antiseizure medication; Drug clearance; Epilepsy; Hormones; Noncompliance; Pregnancy; Seizure control
M. J. Eadie

School of Medicine and Biomedical Science, University of Queensland, Brisbane, QLD 4027, Australia

M. J. Eadie ( $\square)$

4th Floor, Ladhope Chambers, 131 Wickham

Terrace, Brisbane 4000, Australia

e-mail: m.eadie@uq.edu.au 


\section{Key Summary Points}

Most studies have tended to show that seizure control during pregnancy deteriorates more often than it improves.

This deterioration can be explained by (i) patient non-compliance, (ii) failure to adjust antiseizure medication dosages to compensate for the increased drug clearance that occurs in pregnancy, (iii) effects of high circulating female sex hormone concentrations on epileptogenesis, and (iv) the relative inability to use valproate.

Adjusting antiseizure medication dosages to compensate for the increased drug clearance in pregnancy seems to lessen the chance of seizures worsening.

\section{DIGITAL FEATURES}

This article is published with digital features, including a summary slide, to facilitate understanding of the article. To view digital features for this article go to https://doi.org/10.6084/ m9.figshare.14408780.

\section{INTRODUCTION}

For more than half a century there has been an ongoing lack of certainty in the literature as to whether epilepsy tends to become worse during pregnancy, in the sense that existing epileptic seizure disorders become less well controlled. The relevant literature has often not provided a clear-cut answer to the matter, tending to evade doing so by citing the rather consoling observation that published studies have shown that, overall, seizure control during pregnancy usually remains largely unaltered. The present paper attempts to review the available Englishlanguage literature concerning this topic, in the hope of clarifying the situation and discussing various factors that appear relevant to it. The paper is based entirely on previously published data, and contains no material from new unpublished human or animal studies.

\section{THE DEVELOPMENT OF KNOWLEDGE OF SEIZURE DISORDER BEHAVIOUR IN PREGNANCY}

The historical record of human epilepsy extends back over more than 3000 years, but a consideration of the behaviour of epileptic seizure disorders during pregnancy is of very much more recent origin. Over much of the first 100 of the past 250 years during which significantly sized systematic accounts of epilepsy have been available in the medical literature, very little attention was given to the behaviour of epileptic seizures during pregnancy. The topic was not dealt with in Tissot's Traité de l'épilepsie, whose original version first appeared in 1770 [1], or in Pritchard's account [2] from some half a century later. It also escaped notice as a topic in Sieveking's monograph of 1858 [3] and in Russell Reynold's more substantial account 3 years later [4]. These latter two texts were much more highly statistical than the earlier published writings, and their content is more easily relatable to present-day concepts regarding epilepsy. Sieveking and Reynolds were both aware that some women experienced their seizures only at the times of menstruation, and that more often women's seizure frequency increased at this time, i.e. they were aware of what was later termed 'catamenial epilepsy'. However, this knowledge did not seem to lead them in their books to consider what might happen to seizures during pregnancy. In his numerous publications concerning epilepsy a little later in the same century, John Hughlings Jackson [5] also did not consider seizure behaviour during pregnancy, although Jackson's main concern was in interpreting brain function in relation to seizure phenomena.

In the 1901 edition of Gowers' book on epilepsy [6], though not in his earlier 1881 edition, and despite his propensity to quote personally 
recorded statistics, there was merely a statement that, in most cases, women's fits were less frequent during gestation. However, in his first edition [6], Gowers had stated that, in more than $50 \%$ of his 82 cases, epileptic attacks increased around the time of the menses. In contrast to Gowers' view about seizure disorders in pregnancy, his more junior colleague Aldren Turner, in his own 1907 monograph [7], wrote that, in his experience, seizures that had been in remission had recurred during pregnancy in 21 of a series of 61 pregnancies, but also were 'ameliorated' in six.

By that time, there had been at least one publication devoted specifically to the course of seizure disorders during pregnancy, an 1884 University of Paris MD thesis. In it, Raoul Béraud [8] reported that seizures had become more frequent in $26 \%$ of 31 pregnancies, and less frequent in $48 \%$. On that basis he had concluded that pregnancy did not modify the behaviour of epileptic seizure disorders. The thesis did not seem to attract much attention in the contemporary or subsequent literature.

These publications in the late nineteenth and early twentieth centuries provide early examples of the rather contrary conclusions regarding seizure control during pregnancy that continued to be recorded by various authors during the years before the First World War, and during the inter-war period. On the eve of the Second World War, Kinnier Wilson, in his great two-volume textbook of neurology [9], provided references chosen to both support and refute the idea that epilepsy became less well controlled during pregnancy. He took no firm personal position, merely remarking that, from time to time, pregnancy may appear to start the disorder, or induce it to relapse, or arrest or mitigate it.

About three-quarters of the way through the twentieth century, publications began to appear that were concerned explicitly with epilepsy and pregnancy. At much the same time, information became more generally available regarding certain matters potentially relevant to seizure disorder behaviour during pregnancy. These matters included the disposition of certain antiseizure medications in the human body during pregnancy, and the relationships between intra-uterine exposure to various drugs and foetal malformation. As the implications of this information began to be taken into consideration in the later years of the twentieth century, it increasingly influenced those who studied epileptic seizure behaviour during pregnancy, in relation to both the design of their investigations and, particularly, their interpretations of the outcomes.

The first relevant, moderately good-quality data to become available in the past half century were that of Knight and Rhind [10], which appeared in 1975. They reported that seizure frequency had increased during pregnancy in $45.2 \%$ of 153 pregnancies in 59 women, and decreased in only $4.8 \%$. Seven years later, Schmidt [11] combed the available published reports to find that, in a total of 2165 pregnancies in women with epilepsy, seizure frequency had increased in $24.1 \%$ and decreased in $22.7 \%$, with the frequency being unchanged in the remaining $53.2 \%$. In the data sets from the literature that Schmidt had analysed, seizure numbers in pregnancy had increased in 16 individual publications, and decreased in 10 . He was well aware that a variety of criteria for designation of seizure worsening and improvement had been employed in the various studies. A year after that, with colleagues, he published an analysis of 136 pregnancies in 122 women [12]. Seizures increased during pregnancy in 37 of these pregnancies, and decreased in 13. After those publications, increasing numbers of rather better-quality observational studies of the courses of epileptic seizure behaviour during pregnancy began to appear, with the outcomes being analysed employing various criteria that largely reflected the rates of seizure occurrence over various time intervals before and during pregnancy. The outcomes of these and the earlier studies are summarised in Table 1 , without claiming that the collection is exhaustive. The possibility must be acknowledged that the same pregnancy may have been reported more than once, though in different studies. This would particularly be the case in relation to pregnancies in the EURAP [European Registry of Antiepileptic Drugs and Pregnancy] database.

Interestingly, using the individual paper details from the publications in Schmidt's series 
Table 1 Publications dealing with seizure control during pregnancy

\begin{tabular}{|c|c|c|c|c|c|c|}
\hline Author & Year & Number of pregnancies & Number of women & Fewer seizures & & More seizures \\
\hline Bérand [8] & 1884 & 31 & & $48 \%$ & $>$ & $26 \%$ \\
\hline Knight and Rhind [10] & 1975 & 153 & 59 & $4.8 \%$ & $<$ & $45.2 \%$ \\
\hline Schmidt [11] & 1982 & 2165 & & 22.7 & $<$ & $24.1 \%$ \\
\hline Schmidt et al. [12] & 1983 & 136 & 122 & $13 \%$ & $<$ & $37 \%$ \\
\hline $\operatorname{Otani}^{\mathrm{b}}[13]$ & 1985 & & 125 & $4 \%$ & $<$ & $16 \%$ \\
\hline Bardy [14] & 1987 & 154 & 140 & $14 \%$ & $<$ & $32 \%$ \\
\hline Gjerde et al. [15] & 1988 & 78 & 66 & $16.7 \%$ & $=$ & $16.7 \%$ \\
\hline Sabers et al. ${ }^{a}[16]$ & 1998 & 151 & 124 & $7 \%$ & $<$ & $21 \%$ \\
\hline Bag et al. [17] & 1989 & 30 & 30 & $3.3 \%$ & $<$ & $46.7 \%$ \\
\hline Gladstone et al. [18] & 1992 & 9 & 9 & $11.1 \%$ & $<$ & $6.7 \%$ \\
\hline Tanganelli et al. [19] & 1992 & 138 & 97 & $2.9 \%$ & $<$ & $17.4 \%$ \\
\hline Tomson et al. [20] & 1994 & 91 & 70 & $24 \%$ & $>$ & $15 \%$ \\
\hline Thomas et al. [21] & 2001 & 85 & & $9.5 \%$ & $<$ & $12.5 \%$ \\
\hline EURAP Group [22] & 2006 & 1956 & 1882 & $15.9 \%$ & $<$ & $17.3 \%$ \\
\hline Vinnikainen et al. [23] & 2006 & 127 & 85 & $27 \%$ & $>$ & $18 \%$ \\
\hline Battino et al. [24] & 2013 & 3784 & 3451 & $12 \%$ & $<$ & $15.8 \%$ \\
\hline Reisinger et al. [25] & 2013 & 115 & 95 & $17.4 \%$ & $<$ & $38.3 \%$ \\
\hline Cagnetti et al. [26] & 2014 & & 272 & $17.5 \%$ & $<$ & $23.4 \%$ \\
\hline La Neve et al. [27] & 2015 & 56 & & $8 \%$ & $<$ & $19 \%$ \\
\hline Shahla et al. [28] & 2018 & & 94 & $25.5 \%$ & $<$ & $28.7 \%$ \\
\hline Pennell et al. [29] & 2020 & & 351 & $14 \%$ & $<$ & $23 \%$ \\
\hline
\end{tabular}

${ }^{a}$ Results for therapy-compliant women

b Results from the 1971-1987 period. Their earlier results, and at least part of the above one, were probably included in the Schmidt 1982 data

[11] rather than his calculated overall value for his collection, seizures increased during pregnancy in 30 of 44 publications (sign test; $P=0.012$ ).

In the past decade there has been a tendency to evaluate the course of seizure control in pregnancy in relation to another measure, i.e. whether or not the pregnancy concerned was seizure-free as compared with its pre-pregnancy state (and occasionally compared with its postpregnancy one). At least in present-day Western societies, seizure freedom is often a more significant and practically useful consideration than seizure number in determining the impact that experiencing seizures imposes on a woman's way of life, and in particular on her ability to hold a vehicle driving license. In recent times, such seizure freedom usually appears to have been interpreted as freedom from seizures which impair alertness or consciousness rather than total freedom from every perceptible seizure manifestation, though this has not always been made explicit. Several 
studies have included data on seizure numbers as well as on seizure freedom.

For instance, Thomas et al. [30] reported that $82.9 \%$ of their collection of 1281 pregnancies were seizure-free in the pre-pregnancy month, but only $47.8 \%$ remained seizure-free during the ensuing 9 months of pregnancy. Vajda et al. [31] found that $34.5 \%$ of their case series had experienced seizures in the pre-pregnancy year, but $45.9 \%$ suffered seizures during pregnancy: for convulsive seizures the corresponding rates were $18.2 \%$ and $39.4 \%$. La Neve et al. [27], on the other hand, in a much smaller group of subjects $(N=36)$, reported a pre-pregnancy seizure affected rate of $83 \%$ that fell to $72 \%$ during the 9 months of pregnancy. No clear information regarding antiepileptic antiseizure medication use was provided in that paper.

In late 2020, a large-scale multi-centre North American study of seizure occurrence in pregnancy was reported by Pennell et al. [29]. It involved 351 pregnancies in women with epilepsy, and a control population comprising a simultaneously collected and matched 109 nonpregnant women with epilepsy. Seizure control during pregnancy and the following 3 months deteriorated in $23 \%$ of the pregnant women, but also deteriorated in $25 \%$ of the non-pregnant controls. However, in achieving these quite similar outcomes, antiseizure medication therapy had been adjusted substantially more often in the pregnancies (74\% versus $31 \%$ ), the majority of the adjustments involving antiseizure medication dosage increases. The main seizure control comparison made was between during pregnancy and shortly afterwards, and the 9 months following the pregnancy postpartum period, rather than the much more usual comparison between pre-pregnancy and pregnancy. What had happened to the drug therapy in this 9-month post-pregnancy period was not made clear. Thus the main comparison was an after-to-before one, rather than a beforeto-after one. However, a before-to-after pregnancy result was provided in a figure in the paper, and has been used in the data in Table 1 .

The literature material described above could be considered an untidy conglomeration of data derived from non-standardised studies carried out over periods of time that always included pregnancy, or part of it (sometimes the first trimester's seizure occurrence rate served as the comparator for seizures in the subsequent stages of pregnancy), whilst 'pregnancy' in some studies extended into the post-natal weeks. Many of the individual before-to-after comparisons involve seizure occurrence numbers over different periods of time. Antiseizure medication treatment was often changed during pregnancy in the studies, probably mainly to achieve better seizure control. Despite all these limitations for purposes of evaluation, if the collection of data outlined in Table 1 is taken as a whole, it is hard to avoid the impression that seizure control has shown a tendency to worsen during pregnancy. Why should this be so? Several potentially relevant factors need to be considered. They are dealt with immediately below in a general way, rather than in close detail.

\section{FACTORS POTENTIALLY AFFECTING SEIZURE CONTROL DURING PREGNANCY}

\section{Incomplete Compliance with Prescribed Antiseizure Medication Dosages}

Over several decades it has sometimes been suggested that, if epilepsy appeared to become worse in pregnancy, it might simply be due to women failing to take their prescribed dosages of potentially effective antiseizure medications. Such non-compliance might be deliberate or accidental, and be acknowledged or simply not recognised by the women concerned. Several published studies have contained numerical data concerning the extent of the problem. For instance, Shahla et al. [28] reported non-compliance with prescribed therapy in 19\% of the 165 pregnancies they had collected. Increased seizures occurred during pregnancy in $90 \%$ of these non-compliant pregnancies, as compared with a $9.8 \%$ seizure increase rate in pregnancies where women had been compliant. Kusznir Vitturi et al. [32] found evidence of non-compliance with prescribed drug therapy in over $50 \%$ of 82 pregnancies in women with 
treatment-refractory epilepsy; only $3.6 \%$ of these women had seizure-free pregnancies. Other authors such as Otani [13] have indicated that their findings regarding seizure control were obtained only after excluding known noncompliant women, in his case some $27 \%$ of his original patient series. Evidence has been published showing that pre-planning the management of pregnancy in women with epilepsy can result in a reduced incidence of worsened seizure control during pregnancy [33], possibly because such women are more likely to be better informed about the consequences of failing to take prescribed therapy.

\section{Antiseizure Medication Disposition Changes in Pregnancy}

In 1976 Mygind et al. [34], and in the following year Lander et al. [35], reported that the ratio of plasma concentration to the daily dose of the then commonly used antiseizure medications phenytoin and phenobarbitone fell during pregnancy. The change usually began within the first 8 weeks after conception [36]. After these findings became known and their implications appreciated, they were supported by further basically similar investigations which were, in time, extended to the newer antiseizure medications that were increasingly coming into use in pregnant women with epilepsy. The outcome of such studies has been described in a number of publications (e.g. [37-41]).

It appears that, in pharmacokinetic terminology, the body's steady-state clearance (dose/ steady-state plasma concentration) of all the antiseizure medications studied so far has tended to increase during pregnancy. Drugs cleared predominantly or exclusively by renal excretion in unchanged form are lost to the body in increased amounts through the diuresis of pregnancy, which begins quite early. Drugs cleared predominantly by metabolic transformation are increasingly inactivated by the heightened capacity of the liver's drug metabolising enzymes, particularly those that catalyse microsomal oxidation and glucuronidation. This heightened biotransformation capacity is induced by the increased concentrations of circulating female sex hormones that occur during pregnancy. The overall result of the increased drug clearance is that plasma drug concentration relative to drug dose falls during pregnancy, resulting in a decreased pharmacological effect of the drug concerned unless its dose is increased. It has been observed that the clearance of antiseizure medications varies considerably between pregnancies in the same woman [25], while Lander and Eadie [36] noted a tendency for the clearance increase to lessen between the first and subsequent pregnancies in the same woman.

In addition, a physiological fall in plasma protein concentrations occurs in the late stages of pregnancy. This fall causes the measured total plasma drug concentration of any antiseizure medication that is bound to plasma proteins to involve proportionately more unbound, and therefore biologically active, drug substance. Consequently, plasma water drug concentrations may provide a truer measure of drug effect than whole plasma drug concentration measurements, particularly towards the end of pregnancy. However, such unbound drug concentration measurements are not commonly employed in clinical practice.

Obviously this knowledge of the changed antiseizure medication disposition provides a possible explanation for any lessened degree of epileptic seizure control that occurs during pregnancy in women treated with appropriate antiseizure medications taken in the same dosages as before pregnancy. The knowledge also seems likely to have encouraged efforts to achieve better seizure control throughout pregnancy by adjusting antiseizure medication dosages, and this may also have happened even when antiseizure medication concentration monitoring in biological fluids was not carried out.

Accepting a lower circulating antiseizure medication concentration in pregnancy than that which applied previously has been found to be associated with increased seizure occurrence during pregnancy. For instance, Pennell [42] reported that, during pregnancy, allowing the ratio of drug dose to plasma drug concentration of lamotrigine to fall below $65 \%$ of its pre-pregnancy value was associated with 
reduced seizure control. Reisinger et al. [25] also found that a greater than $35 \%$ fall in plasma drug concentration in pregnancy was associated with significant seizure control worsening. Pennell raised the matter again in 2016 [38]. Unfortunately, so far the reported efforts to maintain circulating antiseizure medication concentrations at pre-pregnancy levels have not been entirely successful in rendering pregnancy seizure-free $[36,43]$.

Nevertheless, the attempts over the past three decades to compensate for the antiseizure medication disposition changes mentioned immediately above have probably improved epileptic seizure control during pregnancy substantially. They probably have also resulted in an increased general awareness that over the whole period for which reasonably adequate information on the matter has been available, it usually was not the natural history of epilepsy during pregnancy that was studied, but the behaviour of drug-treated epileptic seizures. Attempting to go back in history to the records of the 1860 s or earlier to try to determine the course during pregnancy of seizure disorders uninfluenced by even modestly effective therapy encounters the difficulty that, since 1857 , an antiseizure medication with definite efficacy has been available in the form of potassium bromide [44, 45], and it was used extensively in the latter half of the nineteenth century.

\section{The Natural History of Untreated Epilepsy During Pregnancy}

While nearly all the available information on seizure behaviour in pregnancy has dealt with drug-treated epilepsy, some of the various case series mentioned in Table 1 did include instances of drug-untreated pregnancies. Unfortunately, the outcomes of these untreated pregnancies usually were not assessed separately but merely included in the overall findings. However, in the set of earlier relevant publications considered in the review by Schmidt [11], there was one series of 108 pregnancies that had not been treated with antiseizure medications. In this series, seizures increased during pregnancy in $22 \%$ but decreased in $39 \%$, the rate remaining unchanged in the remaining 39\%. This information was contained in a 1965 University of Heidelberg dissertation authored by Fuchs [46]. Unfortunately, it has not been possible to readily access it.

The Australian Pregnancy Register data reported by Vajda et al. [31] included 148 pregnancies in women who were not taking antiseizure medications in at least the first half of pregnancy. Some $58.1 \%$ of these pregnancies occurred in women who had either never taken these drugs or had ceased taking them for many months before pregnancy. The remainder had ceased the drugs shortly before becoming pregnant, probably often as a precautionary measure to reduce the risk of foetal malformation, since $56.1 \%$ of this particular subset had resumed drug therapy by the latter part of pregnancy, beyond the stage when foetal malformation would have been initiated. Seizures had occurred in the pre-pregnancy year in $52.2 \%$ of those untreated throughout pregnancy, but in $61.0 \%$ during the 9 months of their pregnancies. There were 39 pregnancies in women with no antiseizure medication exposure for many months or years before pregnancy, and who had been seizure-free in the 12 months before pregnancy. In this subgroup, $35.9 \%$ experienced seizures during pregnancy. All the increases in seizure occurrence recorded in this study were statistically significant. In the following year, in a further analysis of an expanded set of 69 pregnancies in women with untreated epilepsy throughout pregnancy, these authors [47] recorded that $35.5 \%$ had experienced seizures in the pre-pregnancy year, but $56.6 \%$ during the 9 months of pregnancy $(P<0.01)$.

Thus there is some evidence that seizure control that is not influenced by antiseizure medication therapy worsens during pregnancy, and details of the study with a different outcome are not easily obtainable. The possibility therefore cannot be excluded that pregnancy uncomplicated by the effects of antiseizure medication therapy may tend to cause epileptic seizure disorders to become more active during pregnancy. 


\section{Female Sex Hormone Effects}

If pregnancy per se causes seizure disorder control worsening, the role of the female sex hormones and their increased and changing levels throughout pregnancy needs consideration. The literature contains many statements to the effect that oestrogens have a pro-epileptogenic effect, and progesterone an antiepileptogenic one [48]. The existence of catamenial epilepsy correlates with this knowledge, though Joshi and Kuhn [49] have reported the outcome of a study in which progesterone therapy in the luteal phase of the menstrual cycle failed to suppress catamenial seizures in women. A little earlier, Herkes et al. [50, 51] studied daily salivary progesterone concentrations across the menstrual cycle in women with catamenial epilepsy. They found that the premenstrual fall in level tended to correlate with a fall in plasma antiseizure medication concentration relative to dose. This finding raises the possibility that the drug concentration fall may have been the relevant correlate with catamenial seizure occurrence. Nevertheless, it cannot be the full explanation, since the existence of catamenial epilepsy, as mentioned above, was known before the first moderately effective antiseizure medication, potassium bromide, came into use. Further, it was only the subsequently available antiepileptic agents, beginning with phenobarbitone, whose biotransformations were capable of being induced.

Bag et al. [17] looked at the relationship between seizure frequency in pregnancy and circulating sex hormone levels; seizure frequency was increased where oestrogen levels were higher and progesterone ones lower.

\section{Antiseizure Medication Teratogenicity}

The thalidomide episode of the early 1960s alerted the medical community to the possibility that foetal malformation might result from intrauterine exposure to therapeutic drugs taken by the mother. Janz and Fuchs in 1964 [52] found a higher than expected foetal malformation rate associated with antiseizure medication-treated pregnancy, but these authors considered that the association was unlikely to be causal. However, further studies of what were rare events, carried out in relatively small numbers of subjects, began to be reported. These studies continued to raise suspicion that intrauterine exposure to particular antiseizure medications was occasionally responsible for foetal malformation. More definitive information had to await larger-scale studies, nearly all of which were of necessity based on pregnancy registry collections or governmental datasets involving hundreds or even thousands of pregnancies.

Valproate was the first antiseizure medication for which there was reasonably conclusive evidence of teratogenicity. Suspicion began to fall on the drug as early as 1982 [53], and subsequent data from multiple separate sources made it increasingly clear that the drug possessed a significantly greater capacity for causing malformations than the other contemporaneously available antiepileptic agents. Further, its tendency to cause malformations was shown to be related to the drug dose [54, 55].

As time passed, the implication of this knowledge resulted in decreasing valproate use in pregnant women [56]. More recently it has led to virtual prohibition of the use of the drug in the much larger population of women capable of becoming pregnant. Unfortunately, valproate happens to be probably the most effective drug for treating genetic generalised epilepsies, including the relatively common variant juvenile myoclonic epilepsy [57]. Therefore, the increasing inability to employ the drug in practice might have been expected to have unfavourable consequences for epileptic seizure control, both during pregnancy [58] and outside that state. Publications are starting to appear confirming this expectation. Tomson et al., in EURAP data [59], found that valproate had been wholly or partly withdrawn in early pregnancy in 93 pregnancies, withdrawn but replaced by another antiseizure medication in a further 38, and continued unaltered in 1588. The probabilities of generalised convulsive seizures occurring during pregnancy in the three groups were 33\%, 29\% and 16\%, respectively. In 
the experience of Vajda et al. [60], obtained in relation to valproate monotherapy, reduction in pre-pregnancy valproate dosage or cessation of intake of the drug was associated with less than half the risk of foetal malformation compared with pregnancies where valproate intake was continued unchanged ( $4.5 \%$ vs. $10.9 \%)$, but at a cost of a 1.5 times increased hazard of seizureaffected pregnancy (30.8\% vs. $46.2 \%)$.

Whether, and to what extent, this changed use of valproate will affect the neurodevelopment of the offspring of women with epilepsy remains to be seen.

While there is now sufficient evidence to suggest that nearly all of the antiseizure medications currently in common use in pregnant women, except probably levetiracetam, have some potential to cause foetal malformation, their tendencies are distinctly lower than that of valproate. The potential teratogenicity situation regarding more recently marketed antiseizure medications, e.g. lacosamide, perampanel, brivaracetam, is as yet unclear, and is likely to remain so for several years. So far, awareness of the more general teratogenic propensity of the drugs does not seem to have had any appreciable effect on the continuing use of particular antiseizure medications apart from valproate in pregnant women. Regrettably, valproate's hazards have begun to alter, and may further alter, the overall seizure-controlling effectiveness of antiseizure medication therapy in pregnancy.

All the factors considered above, non-compliance, altered drug disposition, hormonal changes altering epileptogenicity, and the teratogenicity hazard that has sometimes resulted in a reluctance to employ the most effective antiepileptic agent in particular women, could contribute to decreased seizure control during pregnancy, each acting in its own right, or in combination with other of the factors. The altered dose to plasma drug concentration relationship, if uncorrected, often may be the major contributor, since the application of a therapeutic policy intended to maintain this relationship at its pre-pregnancy value seems to reduce the risk of seizure disorder worsening during pregnancy. However, the limited data concerning the behaviour of seizure control during pregnancy where there has been no antiseizure medication intake suggests that drug disposition change does not fully account for seizure disorder worsening during pregnancy. The untreated pregnancy data also suggest that incomplete compliance with drug therapy is not the full explanation for seizure disorder worsening during pregnancy. Fortunately, it appears likely that effects of sex hormone changes in relation to seizure disorder worsening in pregnancy can at least sometimes and to some extent be overridden by appropriate antiseizure medication dosage alteration.

\section{RELEVANT RELATED MATTERS}

\section{Seizure Control in Different Types of Epilepsy}

So far in this account, the behaviour of seizures during pregnancy has been considered as if epilepsy was a single homogeneous disease entity. In reality, as Jackson pointed out in 1889 [61], there are 'many different epilepsies', and the individual types may well have different natural histories and different responses in the pregnancy situation. Authors have been aware of this, but in general have not been able to amass sufficient numbers of pregnancies to do more than study the behaviour of antiseizure medication-treated seizure disorders in relation to the frequently occurring entities of genetic generalised and focal epilepsy. This classification of seizure types did not exist until the 1950s, and only became more widely used over the following decade, and the main entities received different and changing designations over that time period.

In 2012, Thomas et al. [30] noted that seizure relapse during pregnancy was more likely in women with focal than in women with generalised epilepsy. Similarly, in the following year, Batttino et al. [24] reported in a larger data set that women with genetic generalised epilepsies were more likely to experience seizure-free pregnancy than those with focal epilepsies $(73.6 \%$ versus $59.5 \%)$. In that same year Reisinger et al. [30], in a series 115 pregnancies in 95 women, also noted that seizures were more likely to occur during pregnancy if the seizure 
disorder was a focal one. Cagnetti et al. [26] reported better seizure control in generalised than in focal epilepsy in their pregnant women with catamenial epilepsy.

In the Vajda et al. data [31] for initially antiseizure medication-untreated epilepsy during pregnancy, $20.6 \%$ of the pregnancies involved were in women who suffered generalised convulsive seizures in the pre-pregnancy year; $26.5 \%$ of their subsequent pregnancies were seizure-affected. In the overall Australian Pregnancy Register data, 37.1\% of the women with genetic generalised epilepsies had seizures during the pre-pregnancy year and 39.6\% during pregnancy, whilst for women with focal epilepsies, the corresponding rates were $49.9 \%$ and $53.6 \%$. Shahla et al. [28] also found a higher risk of loss of seizure control in pregnant women with focal epilepsy.

\section{Predicting Seizure Control During Pregnancy}

Beginning with Vajda et al. in 2008, [62] several investigations have found that the presence or absence of freedom from seizures in the prepregnancy period provides the best predictor of whether the ensuing pregnancy will remain seizure-free. Vajda et al. concluded that seizure freedom in the pre-pregnancy year was their best predictor among the factors that they studied, and also showed that there was little further gain in predictive accuracy from using longer periods of pre-pregnancy seizure freedom (Fig. 1). Harden et al. [63] came to a very similar conclusion in the following year, using the presence or absence of seizure freedom over the 9 months before pregnancy as the predictor. Thomas et al. in 2012 [30] used only the prepregnancy month's seizure control situation as the predictor, again with the same outcome. Several smaller-scale, more recent studies have arrived at basically similar conclusions. For instance, Reisinger et al. [25] noted that 78.8\% of their pregnancies that were seizure-free throughout the pre-pregnancy year remained seizure-free throughout pregnancy, but if seizures had occurred in that year, only $20 \%$ of their pregnancies remained seizure-free.

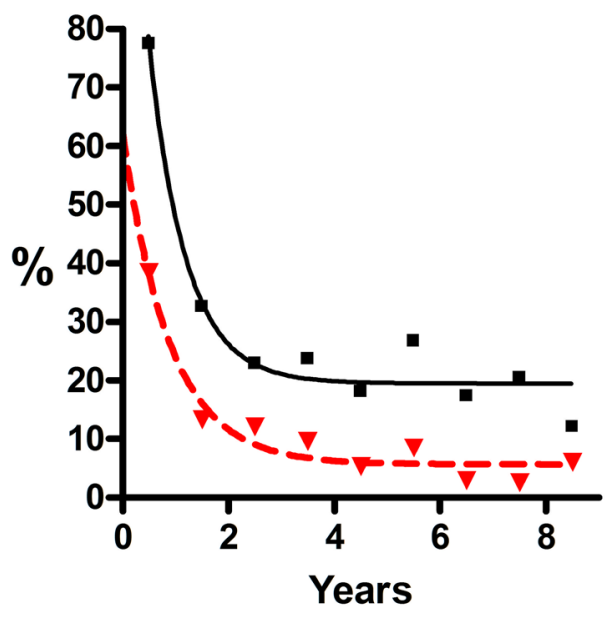

Fig. 1 Rates of occurrence of seizures during pregnancy for any seizure types (black symbols and continuous line) and generalised convulsive seizures (red symbols and broken line) related to duration of seizure freedom before pregnancy. Re-used from Eadie and Vajda [39]

Cagnetti et al. [26] added the finding that having catamenial epilepsy enhanced the possibility of seizure-free pregnancy threefold. Vajda et al. [64] revisited the area in 2018, studying an enlarged data collection from the Australian Pregnancy Register. They then provided evidence that having a seizure disorder not treated with antiseizure medications before pregnancy also increased the hazard of a seizure-affected pregnancy.

Several studies have found that seizure disorders were more likely to worsen during pregnancy if antiseizure medication combinations had been used in treatment $[25,30,65]$, though the roles of the individual drugs in this situation have not been elucidated, and the drug combinations may have been used because the epilepsies in the women involved had not been controlled by antiseizure medication monotherapy.

Vajda et al. [66] examined the matter of seizure control in pregnancy in 44 twin and 2261 singleton pregnancies. They found that the pregnancies were seizure-affected in $34.1 \%$ of the twin ones, but in $45.5 \%$ of the singleton ones $(P>0.05)$. 


\section{DISCUSSION}

The various individual studies on seizure disorders in pregnancy carried out over a century and a half have had many obvious limitations and deficiencies in design, analysis, and interpretation. They have nearly always been based on at least partly uncorroborated evidence that depended on the accuracy of recall of the women who provided the information recorded, or on seizure diaries whose accuracy usually would not have been supported by independent contemporary corroboration. It is likely that seizures that interrupted consciousness for appreciable periods of time, whether or not convulsing occurred, would have been more accurately recorded than seizures with more transient alterations in alertness or behaviour. There was no common standard adopted to determine when seizure occurrence rates were unchanged, and if a policy did exist in a particular study, it often remained unstated. At least one study [27] accepted an up to 50\% alteration in seizure counts as consistent with a lack of change. Such inconsistencies might not invalidate the interpretation of a particular study, but they produce problems in comparing the findings of different studies.

Despite these various limitations of the existing individual published studies, it is difficult to avoid the overall conclusion that epileptic seizure control tends to deteriorate in pregnancy, at least when pre-pregnancy antiseizure medication treatment is not appropriately modified during the course of pregnancy. Yet Stephen et al. (2019) [67] could state, and did so correctly, that 'During pregnancy the majority of women with epilepsy, including those with catamenial epilepsy, have improvement in seizures or remain seizure-free'. That statement is true simply because, in most reports, seizure control remained unchanged in the majority of the pregnancies studied. It is a reassuring statement for women with epilepsy who are either already pregnant or contemplating future pregnancy, and also for those who will manage their pregnancies. However, based on the same data, it would be equally true to assert that the majority of the women with epilepsy who undertake pregnancy have worsened or unaltered seizure control.

If there exists a general tendency for seizure control to deteriorate during pregnancy, why has it not been found in all or nearly all the published investigations? The answer may lie partly in the definition of what constituted unaltered seizure control in particular studies, and partly in the pre-pregnancy drug concentration associated with seizure control in many instances exceeding the concentration needed in the individual to suppress that woman's epileptogenic process. Consequently, if the circulating drug concentration fell during pregnancy, the pre-pregnancy treatment situation had built into it a sufficient margin of safety for the pregnancy drug concentration to remain above the critical threshold for seizure occurrence over the months involved.

\section{CONCLUSIONS}

In view of all the foregoing, from a clinical practice point of view, it would seem that the most sensible policy to adopt in attempting to avoid lost, or further impaired, seizure control during pregnancy would be as follows: to employ an antiseizure medication, or drugs, with the least known potential for teratogenesis and which is appropriate to control the patient's type of seizure disorder; to establish full seizure control for at least several months, and perhaps up to 1 year, before pregnancy begins; and to prescribe appropriate drug dosage adjustments to maintain throughout pregnancy the plasma drug concentrations which applied during the pre-pregnancy period of seizure control. The often provided advice that the prepregnancy drug dose should be the lowest that affords full seizure control, or the best possible seizure control, is rather unrealistic clinically and also arguably unethical, because drug dosage titration to find the threshold dose in the individual will deliberately ensure temporary loss of seizure control with its attendant consequences. Sadly, despite the application of such apparently admirable evidence-based recommendations, the published evidence that is so far available has failed to show that full 
seizure control throughout pregnancy will invariably be achieved by such measures $[29,36]$, perhaps reflecting the limitations of the current therapeutic approach.

\section{ACKNOWLEDGEMENTS}

Funding. No external source of funding was involved in the review's preparation. No funding or sponsorship was received for the publication of this article.

Authorship. The named author meets the International Committee of Medical Journal Editors (ICMJE) criteria for authorship for this article, takes responsibility for the integrity of the work as a whole, and has given approval for this version to be published.

Author's Contributions. The named sole author carried out the literature review, assembled the material selected and wrote the paper without external assistance.

Disclosures. Mervyn J. Eadie has had no perceived conflict of interest in writing this paper.

Compliance with Ethics Guidelines. This review is based entirely on previously published data, and contains no material from new unpublished human or animal studies.

Open Access. This article is licensed under a Creative Commons Attribution-NonCommercial 4.0 International License, which permits any non-commercial use, sharing, adaptation, distribution and reproduction in any medium or format, as long as you give appropriate credit to the original author(s) and the source, provide a link to the Creative Commons licence, and indicate if changes were made. The images or other third party material in this article are included in the article's Creative Commons licence, unless indicated otherwise in a credit line to the material. If material is not included in the article's Creative Commons licence and your intended use is not permitted by statutory regulation or exceeds the permitted use, you will need to obtain permission directly from the copyright holder. To view a copy of this licence, visit http://creativecommons.org/licenses/by$\mathrm{nc} / 4.0 /$.

\section{REFERENCES}

1. Tissot S. Traité de lépilepsie. Paris: Didot de jeune Lausanne, Grasset; 1775.

2. Pritchard JC. A treatise on diseases of the nervous system. Part the first comprising convulsive and maniacal affections. London: Thomas and George Underwood; 1822.

3. Sieveking EH. On epilepsy and epileptiform seizures. London: Churchill; 1858.

4. Reynolds JR. Epilepsy: its symptoms, treatment and relation to other chronic convulsive diseases. London: Churchill; 1861.

5. Taylor J, editor. Selected writings of John Hughlings Jackson. London: Hodder and Stoughton; 1931.

6. Gowers WR. Epilepsy and other chronic convulsive disorders. 1st Edn. 1881, 2nd Edn 1901. London: J \& A Churchill; 1901.

7. Turner WA. Epilepsy. A study of the idiopathic disease. J Nerv Mental Dis. 1907;34:551.

8. Béraud R. De l'épilepsie dans ses rapports avec la grossesse et l'accouchment bromuration pendant la grossesse. Paris: Delahays et Lecrosnier; 1884.

9. Wilson SAK. Neurology. Volume 2. Edited Bruce AN. London. Arnold. 1940.

10. Knight AH, Rhind EG. Epilepsy and pregnancy: a study of 153 pregnancies in 59 patients. Epilepsia. 1975;16:99-110.

11. Schmidt D. The effect of pregnancy on the natural history of epilepsy: review of the literature. In: Janz D, Bossi L, Dam M, Helge H, Richens A, Schmidt D, editors. Epilepsy, pregnancy, and the child. New York: Raven Press; 1982. p. 3-14.

12. Schmidt D, Canger R, Avanzini G, et al. Change in seizure frequency in pregnant epileptic women. J Neurol Neurosurg Psychiatry. 1983;46:751-5.

13. Otani K. Risk factors for the increased seizure frequency during pregnancy and puerperium. Folia Psychiatr Neurol Jpn. 1985;39:33-41. 
14. Bardy AH. Incidence of seizures during pregnancy, labor and the puerperium in epileptic women: a prospective study. Acta Neurol Scand. 1987;75: 356-60.

15. Gjerde IO, Strandjord RE, Ulstein M. The course of epilepsy during pregnancy: a study of 78 cases. Acta Neurol Scand. 1988;78:198-205.

16. Sabers A, Rogvi-Hansen B, Dam M, et al. Pregnancy and epilepsy: a retrospective study of 151 pregnancies. Acta Neurol Scand. 1998;97:164-70.

17. Bag S, Behari M, Ahuja GK, Karmarkar MG. Pregnancy and epilepsy. J Neurol. 1989;236:311-3.

18. Gladstone DJ, Bologa M, Maguire C, Pastuszak A, Koren G. Course of pregnancy and fetal outcome following maternal exposure to carbamazepine and phenytoin: a prospective study. Reprod Toxicol. 1992;6:257-61.

19. Tanganelli P, Regesta G. Epilepsy, pregnancy, and major birth anomalies: an Italian prospective, controlled study. Neurology. 1992;42(4 Suppl 5):89-93.

20. Tomson T, Lindbom U, Ekqvist B, Sundqvist A. Epilepsy and pregnancy: a prospective study of seizure control in relation to free and total plasma concentrations of carbamazepine and phenytoin. Epilepsia. 1994;35:122-30.

21. Thomas SV, Indrani L, Devi GC, et al. Pregnancy in women with epilepsy: preliminary results of Kerala registry of epilepsy and pregnancy. Neurol India. 2001;49:60-6.

22. The EURAP Study Group. Seizure control and treatment in pregnancy. Observations from the EURAP Epilepsy Pregnancy Registry. Neurology. 2006;66:354-60.

23. Viinikainen K, Heinonen S, Eriksson K, Kälviäinen R. Community-based, prospective, controlled study of obstetric and neonatal outcome of 179 pregnancies in women with epilepsy. Epilepsia. 2006;47:186-92.

24. Battino D, Tomson T, Bonizzoni E, et al. Seizure control and treatment changes in pregnancy: observations from the EURAP epilepsy pregnancy registry. Epilepsia. 2013;54:1621-7.

25. Reisinger TL, Newman M, Loring DW, Pennell PB, Meador KJ. Antiepileptic grug clearance and seizure frequency during pregnancy in women with epilepsy. Epilepsy Behav. 2013;29:13-8.

26. Cagnetti C, Lattanzi S, Foschi N, Provinciali L, Silvestrini $M$. Seizure course during pregnancy in catamenial epilepsy. Neurology. 2014;83:339-44.
27. La Neve A, Boero G, Francavilla T, Plantamura M, De Agazio G, Specchio LM. Prospective, case-control study on the effect of pregnancy on seizure frequency in women with epilepsy. Neurol Sci. 2015;36:79-83.

28. Shahla M, Hijran B, Sharif M. The course of epilepsy and seizure control in pregnant women. Acta Neurol Belg. 2018;118:459-64.

29. Pennell PB, French JA, May RC, et al. Changes in seizure frequency and antiepileptic therapy during pregnancy. N Engl J Med. 2020;383:2547-56.

30. Thomas SV, Syam U, Devi JS. Predictors of seizures during pregnancy in women with epilepsy. Epilepsia. 2012;53:e85-8.

31. Vajda FJ, O'Brien TJ, Graham J, Lander CM, Eadie MJ. The outcomes of pregnancy in women with untreated epilepsy. Seizure. 2015;24:77-81.

32. Kusznir Vitturi BK, Cabral FB, Mella CC. Outcomes of pregnant women with refractory epilepsy. Seizure. 2019;69:251-7.

33. Abe K, Hamada H, Yamada T, Obata-Yasuoka M, Minakami H, Yoshikawa H. Impact of planning of pregnancy in women with epilepsy on seizure control during pregnancy and on maternal and neonatal outcomes. Seizure. 2014;23:112-6.

34. Mygind KI, Dam M, Christiansen J. Phenytoin and phenobarbitone plasma clearance during pregnancy. Acta Neurol Scand. 1976;54:160-6.

35. Lander CM, Edwards VE, Eadie MJ, Tyrer JH. Plasma anticonvulsant concentrations during pregnancy. Neurology. 1977;27:128-31.

36. Lander CM, Eadie MJ. Plasma antiepileptic drug concentrations during pregnancy. Epilepsia. 1991;32:257-66.

37. Pennell PB, Gidal BE, Sabers A, Gordon J, Perucca E. Pharmacology of antiepileptic drug during pregnancy and lactation. Epilepsy Behav. 2007;11: 263-9.

38. Pennell PB. Use of antiepileptic drugs during pregnancy: evolving concepts. Neurotherapeutics. 2016;13:811-20.

39. Eadie MJ, Vajda FJE. Antiepileptic drugs and pregnancy. Heidelberg: Springer; 2016.

40. Voinescu PE, Pennell PB. Management of epilepsy during pregnancy. Expert Rev Neurother. 2015;15: 1171-87.

41. Tomson T, Battino D, Bromley R, et al. Management of epilepsy in pregnancy: a report from the 
International League Against Epilepsy Task Force on Women and Pregnancy. Epilept Disord. 2019;21:497-517.

42. Pennell PB, Peng L, Newport DJ, et al. Lamotrigine in pregnancy: clearance, therapeutic drug monitoring, and seizure frequency. Neurology. 2008;70: 2130-6.

43. Thangaratinam S, Marlin N, Newton S, et al. Antiepileptic drug Monitoring in PREgnancy (EMPiRE): a double-blind randomised trial on effectiveness and acceptability of monitoring strategies. Health Technol Assess. 2018;22:1-152.

44. Locock C. Discussion of paper by E H Sieveking. Analysis of fifty-two cases of epilepsy observed by the author. Lancet. 1857;40:527-8.

45. Wilks S. Bromide and iodide of potassium in epilepsy. Med Times and Gaz. 1861;72:635-6.

46. Fuchs U. Epilepsie und Schwangerschaft. Inaugural Dissertation. 1965. Heidelberg. Cited by Schmidt D (1982) Reference 11.

47. Vajda FJ, O'Brien TJ, Lander CM, Graham J, Eadie MJ. Does pregnancy per se make epilepsy worse? Acta Neurol Scand. 2016;133:380-3.

48. Taubøll E, Sveberg L, Svalheim S. Interactions between hormones and epilepsy. Seizure. 2015;28: 3-11.

49. Joshi S, Kapur J. Neurosteroid regulation of $\mathrm{GABA}_{\mathrm{A}}$ receptors: a role in catamenial epilepsy. Brain Res. 2019;1703:31-40.

50. Herkes GK, Eadie MJ. Possible roles for frequent salivary antiepileptic drug monitoring in the management of epilepsy. Epilepsy Res. 1990;6:146-54.

51. Herkes GK, Eadie MJ, Sharbrough F, Moyer T. Patterns of seizure occurrence in catamenial epilepsy. Epilepsy Res. 1993;15:47-52.

52. Janz D, Fuchs U. Sind antiepileptische medikamente waehrend der schwangerschaft schaedlich? [are anti-epileptic drugs harmful during pregnancy?]. Dtsch Med Wochenschr. 1964;89:241-8.

53. Robert E, Guibaud P. Maternal valproic acid and congenital neural tube defects. Lancet. 1982;2:937.

54. Vajda FJ, Eadie MJ. Maternal valproate dosage and foetal malformations. Acta Neurol Scand. 2005;112: 137-43.
55. Kaneko S, Battino D, Andermann E, et al. Congenital malformations due to antiepileptic drugs. Epilepsy Res. 1999;33:145-58.

56. Vajda FJ, Horgan D, Hollingworth S, et al. The prescribing of antiepileptic drugs for pregnant Australian women. Aust N Z J Obstet Gynaecol. 2012;52:49-53.

57. Serafini A, Gerard E, Genton P, Crespel A, Gelisse P. Treatment of juvenile myoclonic epilepsy in patients of child-bearing potential. CNS Drugs. 2019;33:195-208.

58. Tomson T, Marson A, Boon P, et al. Valproate in the treatment of epilepsy in girls and women of childbearing potential. Epilepsia. 2015;56:1006-19.

59. Tomson T, Battino D, Bonizzoni E, et al. Withdrawal of valproic acid treatment during pregnancy and seizure outcome: observations from EURAP. Epilepsia. 2016;57:e173-1737.

60. Vajda FJE, O'Brien TJ, Graham JE, Hitchcock AA, Lander CM, Eadie MJ. Pregnancy after valproate withdrawal-Fetal malformations and seizure control. Epilepsia. 2020;61:944-50.

61. Jackson JH. Remarks on evolution and dissolution of the nervous system. J Mental Sci. 1887;33:25-48.

62. Vajda FJE, Hirchcock A, Graham J, O'Brien T, Lander C, Eadie M. Seizure control in antiepileptic drug-treated pregnancy. Epilepsia. 2008;49:172-5.

63. Harden C, Hopp J, Ting TY, et al. Management issues for women with epilepsy - Focus on pregnancy (an evidence-based review): 1. Obstetrical complications and change in seizure frequency. Epilepsia. 2009;50:1229-36.

64. Vajda FJE, O'Brien TJ, Graham JE, Hitchcock AA, Lander CM, Eadie MJ. Predicting epileptic seizure control during pregnancy. Epilepsy Behav. 2018;78: 91-5.

65. EURAP Study Group. Seizure control and treatment in pregnancy: observations from the EURAP epilepsy pregnancy registry. Neurology. 2006;66: 354-60.

66. Vajda FJE, O'Brien TJ, Graham JE, et al. Twin pregnancy in women with epilepsy. Epilepsia. 2020;61: 2748-53.

67. Stephen LJ, Harden C, Tomson T, Brodie MJ. Management of epilepsy in women. Lancet Neurol. 2019;18:481-91. 